\title{
Development of a Common Software Interface
}

\author{
B. Davis \\ J. Parrett
}

June 16, 1997

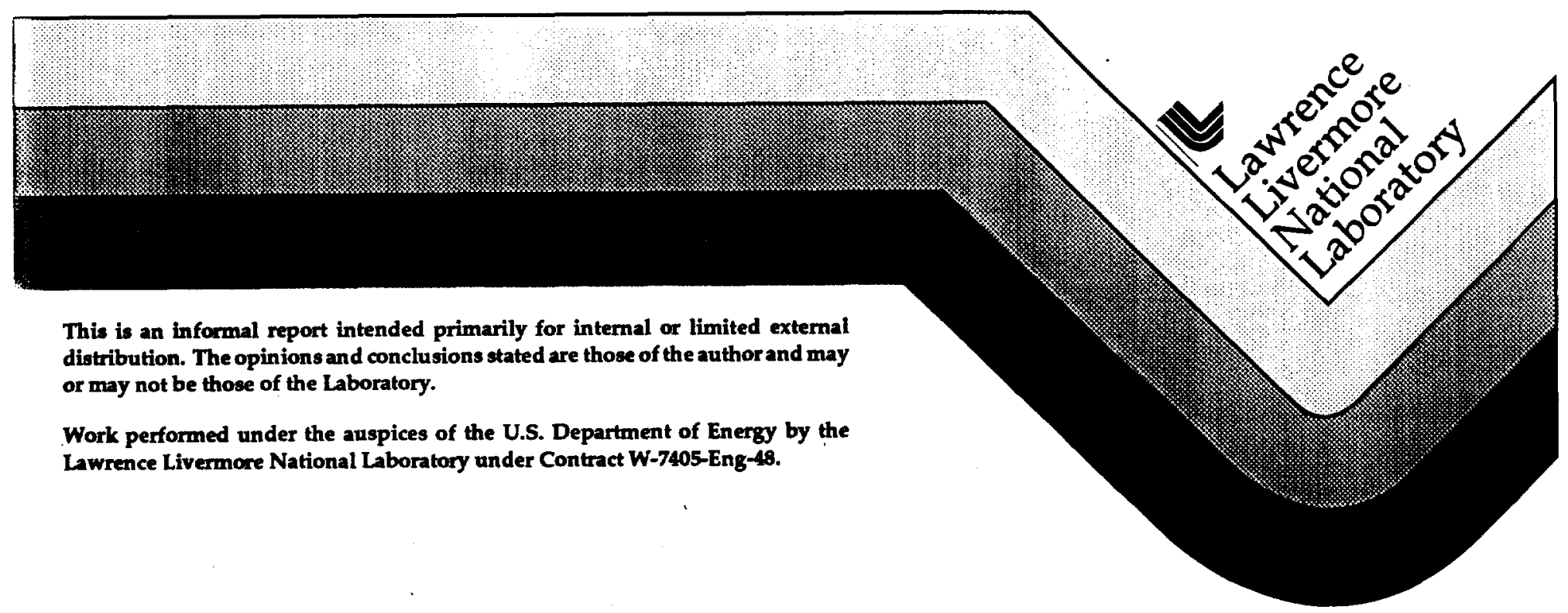




\section{DISCLAIMER}

This document was prepared as an account of work sponsored by an agency of the United States Government. Neither the United States Government nor the University of California nor any of their employees, makes any warranty, express or implied, or assumes any legal liability or responsibility for the accuracy, completeness, or usefulness of any information, apparatus, product, or process disclosed, or represents that its use would not infringe privately owned rights. Reference herein to any specific commercial product, process, or service by trade name, trademark, manufacturer, or otherwise, does not necessarily constitute or imply its endorsement, recommendation, or favoring by the United States Government or the University of California. The views and opinions of authors expressed herein do not necessarily state or reflect those of the United States Government or the University of California, and shall not be used for advertising or product endorsement purposes.

This report has been reproduced directly from the best available copy.

Available to DOE and DOE contractors from the Office of Scientific and Technical Information

P.O. Box 62, Oak Ridge, TN 37831

Prices available from (615) 576-8401, FTS 626-8401

Available to the public from the

National Technical Information Service

U.S. Department of Commerce

5285 Port Royal Rd.,

Springfield, VA 22161 


\title{
Development of a Common Software Interface
}

\author{
Information Technology and Security Center
}

\section{Executive Summary}

The variety of applications, databases, and information technologies makes it difficult to locate and retrieve information or applications that are otherwise openly available. The promise of the World Wide Web was that it was a paradigm for organizing and distributing information. Under this project, we looked at how we could more tightly couple the Web with the UNIX operating system for exchanging information, connecting programs and data, and facilitating collaboration between people in a more natural way. Ease of secure access and the ability to have electronic dialogues on specific questions will become increasingly important as the pressure increases for scientists and analysts to rapidly access diverse information to make informed judgments and initiate new lines of investigation.

\subsection{Background}

The World Wide Web, henceforth called the Web, and Web-browsing tools have shown their value in providing an easy-to-use interface to various types of data, such as text, audio, and video. In this project we are exploring the potential of the Web browsers as a common software interface (CSI) to provide a single interface paradigm to the diverse computing environments and services (scientific applications and databases, collaborative dialogue, and collaborative work environments), along with the machine time needed to run them.

Under this Laboratory Directed Research and Development (LDRD) project, we focused on the humansystems issues of collaboration and the information security issues, including availability, confidentiality, and integrity - specifically from the perspective of "participatory decision-making." The project leveraged the abilities of the hypertext transport protocol (http) to give users a simple point-and-click interface to find and connect to applications and databases, supplemented by a collaborative workspace organized around a focused electronic discussion on relevant issues. The security decision-making focus required the exploration of the use of a digital signature, access control to information, and protection of proprietary information and the audit and archiving of information generated in support of decision making. In keeping with the decision-making focus, the development of Web-based collaborative tools (electronic forums, electronic chats) was required. The connection to the applications, databases, and collaborative forums was handled through a modular Web browser CSI. For example, an analytical application is chosen using point-and-click; transparent to the user, an external code causes the X protocol to present the requested application on the user's (client) computer. The user then interacts with the application's native interface or via a Web form. In some cases, the analytical application's input and output can be handled completely under the Web, and external viewers are not required. In both cases an archive record to the session is then generated and displayed for the decision-maker, who can sign digitally to authenticate the information.

This scheme of a modular browser (composed of independent external programs, processes, or threads) allows each subcomponent to be optimized for the interaction with the particular application or database. An implicit benefit of this approach is that it also allows for some parallelism. The Web is based on full autonomy of the server; information processing from the server is done locally on the client. This modular Web browser as a CSI introduces parallelism, as the external codes are commanding the server, or distributed servers, to perform additional processing beyond the synchronous nature of the Web. In addition, through this scheme of external programs, authentication and access control to individual 
applications or databases can be performed, allowing users to execute only the services for which they are authorized while logging usage of compute cycles and disk space.

\section{CSI Capabilities}

An objective of the CSI project was to develop and use Web-based electronic communications technology as a basis for focused collaboration and participatory decision-making. The interface was required to interact with information/data/programs and support dialogue on the same.

The CSI project had three thrust areas. The first was exploring the use of a Web-browser to allow access to programs or applications, and allow a user to interact with those programs or applications. The second was to develop an application for focused dialogue leading toward decision making. The third was to explore the human-systems problem associated with electronic collaboration. This CSI project lead to the development of three Web-based pieces of collaborative software. They are NetForum, NetDoc, and GEn\&SIS. NetForum provides a virtual discussion space where participants can interact over the Internet using the Web, and electronic mail technology. It uses open systems standards and concepts to provide a suite of capabilities. NetFonum is focused on providing users a threaded discussion space on an issue. NetDoc uses NetForum to provide a threaded discussion space anchored to a document being reviewed. GEn\&SIS uses a graphical information system (GIS) and the Web to display geographical information spatially. It is meant to be a modular interactive system that will provide the ability to view site and environmental licensing documents, search for and retrieve remote information, and use GIS as a display engine to view geographic, geologic, etc. information as part of the site review and assessment process. Via a Web-browser, permitted users can gain access, specify a site within the U.S., choose the geographical data sets of interest, and display a map of the site and selected information. GEn\&SIS also links to seismic hazard analysis codes and the data sets which support the codes. Permitted users may link to the analysis codes and run the appropriate application for their selected location GEn\&SIS must provide for information integrity, security, and control.

\subsection{HTML Usage}

Hypertext markup language (HTML) is a simple markup language used to create hypertext documents that are portable from one platform to another. HTML documents are standard general markup language (SGML) documents with generic semantics that are appropriate for representing information from a wide variety of applications. SGML is considered to be a complex but mature, stable technology. HTML is a simple, new, and rapidly evolving language which is used to construct documents that can be viewed by Web browsers. HTML has been standardized by the World Wide Web Consortium $\left(W^{3} \mathrm{C}\right)$ in Internet Engineering Task Force (IETF) Request for Comment (RFC) 1866, commonly referred to as HTML Version 2. The IETF is a set of working groups under the Internet Society. IETF RFC 1866 forms the basis for further work in extending and enhancing the standard, and various user agent vendors are already shipping products with extensions beyond this RFC. RFC 1866 also declares some earlier HTML elements obsolete. Work ongoing to standardize specifications of many enhancements to RFC 1866 on a wide variety of topics. These topics are described below.

\subsubsection{APPLET and EMBED}

Work is ongoing to merge these two mechanisms to invoke/include some non-HTML material on an HTML page. The intent is to allow applications like JAVATM to be used, and also to develop a much more generalized HTML markup mechanism. This topical area will change dramatically in the near future. 


\subsubsection{Client-Side Image Maps}

This is to define areas of an IMG which, if selected, will invoke identified hyperlinks. This capability already exists by placing an IMG with an ISMAP attribute within the contents of an element which points to a program which will accept the coordinates selected and take action. This proposal is to define the attributes that will allow all this to be specified in the actual HTML document without need for a responding program. A proposal exists in this area, but further work is not apparent at this time even though many browsers are implementing enhancements.

\subsubsection{Form-Based File Upload in HTML}

File-upload is a feature that benefits many applications. RFC 1867 proposes extending HTML to allow information providers to express file upload requests uniformly, and a MIME-compatible representation for file upload responses. RFC 1867 also includes a description of a backward-compatibility strategy that allows new servers to interact with current HTML user agents. More work needs to be done in this area to merge RFC 1867 with RFC 1866.

\subsubsection{Internationalization of HTML}

This involves HTML elements and attributes to ease the use of non-English languages in Web pages. Note that this project must address not only the problem of right-to-left languages but also non-English alphabets/scripts. A foremost consideration is to make sure that HTML remains a valid application of SGML, while enabling its use in all languages of the world. This is commonly referred to as the i18n proposal.

\subsubsection{Links}

Link semantics and links to frozen documents are two key issues. Links with semantics have been in the specifications for a long time. The concept is to have Web clients that can edit the semantics, and then machines to analyze a semantic web. The topic of REI REV must also be dealt with. Linking to frozen documents refers to data that is not marked up, which means that links to it must be made to the document URL only. The proposal is to use some sort of coordinate system to define an anchor. Work in this area seems to be slow to come to a proposal, but a low level of discussion continues.

\subsubsection{Mathematical Equations}

The proposal for HTML+ covers representing equations directly in HTML using an SGML-based notation. The proposal indicates that the handling of mathematical equations is inspired by the approach taken by LaTeX. A experimental browser supporting the MATH element is being developed by CERN. The effort to date has been focused on resolving the majority of users' needs, rather than aiming for complete coverage. At present users are including mathematical equations as images.

\subsubsection{Style Sheets}

A draft cascading style sheet mechanism has been proposed $\left(\mathrm{W}^{3} \mathrm{C}\right.$ Working Draft, Feb. 20, 1996). While some of the implementation details are still in hot debate, the basic concept of providing some linkage from an HTML page to style sheets to specify presentation is completely accepted. The concept of a style sheet is to allow authors and readers to attach style (e.g. fonts, colors and spacing) to HTML documents. Most of the draft specification is focused on the rules for resolving conflicts between an author's preferred style sheet and a reader's personal style sheet. It is fairly safe to assume that most vendors will let the standards group get closer to final agreement before they begin to offer this capability. 


\subsubsection{HTML3 Table Model}

The $\mathrm{W}^{3} \mathrm{C}$ has come to a consensus and developed a draft specification to extend HTML to support a wide variety of tables. It includes significant changes and enhancements from existing implementations of tables, yet does not go as far in providing table layout and presentation control as some wanted. The HTML table model is designed to work well with associated style sheets, but does not require them. The table model uses certain aspects of the CALS table model, e.g., the ability to group table rows into thead, tbody and tfoot sections, plus the ability to specify cell alignment compactly for sets of cells according to the context. Future enhancements to the area of tables are expected.

As alluded to in Section 2.1.6, some of these enhancements were packaged earlier as an HTML+ proposal (November 1993) and as a proposal known as HTML 3.0 (March 1995). The HTML 3.0 proposal encompassed much of what was in the HTML+ proposal and extended it. The proposals have been split into separate topics for ease of discussion in the standards group, and the package known as HTML 3.0 has been withdrawn. HTML+ was a preliminary draft of the proposals known as HTML 3.0. There is now some talk of interim HTML 2.x to standardize the various separate topics as they reach consensus. It is unclear when, or if, there will be a sufficient collection of enhancements to be (again) called HTML 3.0.

\subsection{Security Issues}

There are two givens in the software development world. The first given is that buggy software opens up security holes. The second given is that large, complex programs contain bugs. Both the Web server software and the Web client software are large, complex codes and have already been proven to contain security holes. Security issues are intensified by the very nature of the Web-an open architecture developed for the sharing of information. Most Web servers reside on UNIX platforms, and the UNIX operating system falls under the heading of a large, complex program. UNIX is a very powerful, flexible operating system and thus suffers from bugs. The flexibility of the operating system can lead to configurations that leave the computer system open to attack. It must be noted that integration of security into the services being provided should be an underlying theme in the development of all information systems. The Information Technology and Security Center (IT\&S) tries to take a graded approach to information security and the development of information systems. To that end IT\&S uses a risk analysis matrix to identify information security needs in the planned information system. The process starts by defining the functionality desired and the information system requirements, including action plans. The next step is to develop cost estimates associated with the action plans (cost/benefit analysis). From this a determination is made as to the level of risk the organization is willing to assume. With that determination, an implementation plan is developed. The key issue to determining the level of risk an organization is willing to assume is by defining in organizational terms the impact of undesirable or unauthorized events on organizational data.

The IT\&S in general separates Web security issues into two areas: host security issues and software security issues. Web security issues are categorized as shown in Table 1. 
Table 1. Web Security Issues

\begin{tabular}{|c|c|}
\hline Host Security & Software Security \\
\hline $\begin{array}{l}\text { Firewall-First line of defense. Not by any means a } \\
\text { guarantee of security, but it will buy time in case of a } \\
\text { break-in, and make breaking in much more difficult. The } \\
\text { use of a firewall is determined by the risk assessment. } \\
\text { Patches-Generic operating systems as released contain } \\
\text { MANY security holes. Patches must be applied } \\
\text { periodically. } \\
\text { No remote root logins-Require privileged users to "su" } \\
\text { to root; this results in an accounting of what login is } \\
\text { attempting to become root. }\end{array}$ & $\begin{array}{l}\text { Server Software-Some } \\
\text { are more secure than } \\
\text { others; from NCSA use } \\
\text { Version } 1.4 \text { or higher. } \\
\text { CERN and Apache are } \\
\text { other UNIX-based free } \\
\text { server software. In } \\
\text { general, the more features } \\
\text { a server offers, the more } \\
\text { security holes it is apt to } \\
\text { contain. }\end{array}$ \\
\hline $\begin{array}{l}\text { One time/SKey passwords-Use of "sniffed" passwords } \\
\text { to gain access to the machine can be avoided by the use of } \\
\text { one-time passwords. If not using one-time passwording, } \\
\text { then make users practice good passwording, and audit } \\
\text { periodically. A program called "SPY" can help accomplish } \\
\text { this. } \\
\text { Services-Removal of unused/vulnerable services can } \\
\text { close many security holes. This includes the removal of } \\
\text { unneeded shells and interpreters. }\end{array}$ & $\begin{array}{l}\text { Access.conf-Make sure } \\
\text { the server is configured } \\
\text { correctly; access.conf is } \\
\text { the backbone of the Web } \\
\text { server software security, } \\
\text { assuming that the software } \\
\text { itself is secure. }\end{array}$ \\
\hline $\begin{array}{l}\text { Wrappers (TCP, CGI) Place "wrappers" on required } \\
\text { services; this is an accountability/restriction tool, as well as } \\
\text { a source of warnings about potential problems. } \\
\text { Tripwire-In case of a break-in, tripwire will allow the } \\
\text { system administrator to easily find out what files have been } \\
\text { tampered with. As a minimum, regularly check the system } \\
\text { and Web logs. } \\
\text { File Permissions-Make sure that ALL files accessible to } \\
\text { the httpd server are not writable by "world." A program } \\
\text { called "COPS" can help accomplish this. }\end{array}$ & $\begin{array}{l}\text { Client Software-In } \\
\text { general, beware of using } \\
\text { external viewers for any } \\
\text { file that contains } \\
\text { executable statements. } \\
\text { This security issue is } \\
\text { supposed to be addressed } \\
\text { by scripting languages } \\
\text { Java }{ }^{\mathrm{TM}} \text { and Safe Tcl } \\
\text { There } \\
\text { Safe Perl } \\
\text { SMen a prototype }\end{array}$ \\
\hline $\begin{array}{l}\text { user logins-The less access given to this machine, the } \\
\text { ver possibilities there are for a break-in. } \\
\text { ndmail-Don't run it if possible. If it is necessary, use } \\
\text { ndmail version } 8.6 .10 \text { or higher, and make sure it is } \\
\text { nfigured correctly. Don't use the general distribution } \\
\text { admail program. }\end{array}$ & $\begin{array}{l}\text { cgi programs-Use Perl } \\
\text { or C; Shell scripts are } \\
\text { known to be notorious } \\
\text { entry points for break-ins } \\
\text { when run under http. }\end{array}$ \\
\hline $\begin{array}{l}\text { FTP_-Don't run FTP if possible. If it is necessary, use } \\
\text { more secure FTP server software than the distribution. } \\
\text { Washington University-St. Louis FTP server version } \\
2.4 \text { _3 is a good replacement. }\end{array}$ & $\begin{array}{l}\text { Run as root?-Having } \\
\text { the server run as an } \\
\text { unprivileged user may } \\
\text { avoid some security } \\
\text { problems. }\end{array}$ \\
\hline
\end{tabular}


The risk analysis matrix is basically a formal process used to analyze what risks or events the data needs to be protected from. It becomes a method to formalize security objectives. Security objectives are generally defined in terms of the following:

- Integrity-Unauthorized or undesirable modification or destruction of data, information, or system services.

- Confidentiality-Unauthorized or undesirable disclosure of data, information or system services.

- Availability-Unavailability of data or system services.

Security risks fall under two general categories:

- Accidental acts-undesirable events; errors and omissions.

- Deliberate acts-unauthorized events; fraud and misuse.

As can be seen, the above basically define the risk analysis matrix shown below. What falls out of the risk analysis are examples of threats and examples of controls.

Table 2. Information Security Risk Analysis Matrix

\begin{tabular}{|c|c|c|c|c|}
\hline & & DATA & & \\
\hline & Integrity & Confidentiality & Availability & \\
\hline $\begin{array}{l}\text { Accidental } \\
\text { acts }\end{array}$ & & & & $\begin{array}{l}\text { Undesirable event } \\
\text { (Enor \& omission) }\end{array}$ \\
\hline $\begin{array}{l}\text { Deliberate } \\
\text { acts }\end{array}$ & & & & $\begin{array}{l}\text { Unauthorized event } \\
\text { (Fraud \& misuse) }\end{array}$ \\
\hline & $\begin{array}{l}\text { Modification or } \\
\text { destruction of } \\
\text { information }\end{array}$ & $\begin{array}{l}\text { Disclosure of } \\
\text { information }\end{array}$ & $\begin{array}{l}\text { Unavailability } \\
\text { of information } \\
\text { or service }\end{array}$ & \\
\hline
\end{tabular}

\subsection{Authentication Issues}

The following are necessary for the Web to become an environment within which people can collaborate, not just a conduit for finding information.

- Resource organization-Tools are needed to organize the information/data/programs in workspaces which reflects the way a group or team of people work and perceive the information they need to share.

- Access control-For secure collaboration to occur through the Web, a security kernel needs to be in place which provides access control in a robust and transparent way. The access control will be responsible for allowing access not only to data but also to compute cycles on machines.

- Pipes across machines-A trusted system is needed to take care of authenticating privileges across trusted systems and achieve the necessary networking. 
- Writing files-As teams collaborate, and use the Web to launch and run programs, the need will arise to write the output on local disks. A mechanism needs to be in place that allows trusted parties to do this.

The most logical way to meet these needs is through the use of public key cryptography (PKC). PKC enables the communicating parties to authenticate each other without sharing secret information. ITU X.509 Standard type certificates provide identity of an entity (user, process, etc.). Some kind of encapsulation of authority needs to be created. Under the LDRD, encapsulation of authority was accomplished by including an additional field with the certificate which contains some authority string. Both the identity information and the authorization information must be signed to prevent forgery.

Most transactions involve more than two parties from start to finish. Take for example a credit card charge. A buyer (party 1) wants to make an electronic purchase from an online merchant (party 2). The merchant wants to verify the credit card number and see if the buyer has sufficient credit available. This verification is typically done using credit card authorization companies (party 3). As this transaction progresses, information from party 1 is passed to party 2, who then interacts with party 3, passing an authorization certificate back to party 2 . Party 2 then completes the transaction by providing party 1 with some type of receipt certificate.

Most certificate models are based on a static structure, which works fine for identity information that is relatively static (although there is a problem with how to implement revocation lists). Authorization information, on the other hand, can be very dynamic, as shown in the previous example. To support authorization, some type of real-time mechanism for generating short-lived certificates is needed.

Finally, the generation of some type of receipt which captures the results of a transaction is necessary for record-keeping and in case of legal challenges. This receipt would look very much like a certificate with a few more fields.

Under the LDRD, the idea was to use a protocol which could be incorporated into the Web; this protocol would encode information into the request and the result would be a certificate which contains the result of the request. This transaction access protocol (TAP) would be very similar to HTTP in the sense that it could include static information along with transaction-specific information.

In the credit card example, a consumer makes a request to his or her credit card company for a certificate that allows him or her to perform electronic purchases. It might contain the following information:

Identity: John B. Doe

Public Key: $x \times x \times x \times x \times x \times x \times x x$

Authorization: /visa/Bankname/electronic/purchase/xxxx-yyyy-zzzz

Signed: aaaaaaaaaaaaaaaaaaa

The identity is just like a distinguishing name in a X.509 certificate. The public key was what was generated or provided when the consumer requested this certificate from Bankname visa. The authorization string contains what the consumer is requesting to be able to do with this certificate-note that the $x x x x$-yyyy-zzzz is the credit card number for the Bankname visa. This authorization string is what must be presented along with the public key to perform an electronic transaction. The signed string was generated by Bankname visa when they granted this authority. Note, only the public key and authorization are signed. The identity could be blank in a situation where anonymous transactions are desired.

Now that the consumer has been granted authority, he or she visits the widgets Web site intending to purchase some widgets. The consumer fills out the on-line form, signs it with the private key and includes the authorization certificate. Widgets Inc. has established a relationship with an on-line credit card 
verifier that has authorized them to do credit-check. When they get the request they use TAP to make an authorization request. The authorization request might look like this:

TAP::/online.verify.com/credit-check?

authorization=visa/Bankname/electronic/purchase/ $x x x x-y y y y-z z z z \&$ publickey $=x x x x x$

$x \times x \times x \times x \times x$ \&signed"aaaaaaaaaaaaaaaa\&\&fom=Widgets Inc.\&amount_0.00

Note the similarity to URL encoding used for HTTP with the submission of form data. The basic structure for TAP is: TAP:/host/authorization?transaction data. This allows static information to be encoded along with the dynamic information. The exact content of the dynamic information is based on the type of transaction being executed. It would probably be necessary for Widgets Inc. to sign the request. The result of this request would be a receipt like this:

Identity: online.verify.com

Public Key: xyxyxyxyxyxyxyxy

Authorization/visa/Bankname/electronic/purchase/xxxx-yyyy-zzzz

Key: $\mathrm{xxxxxxxxxxxxx}$

From: Widgets Inc.

Status: Approved

Signed: mnmnmnmnmnmnmmn

This would represent a signed certificate which Widgets Inc. could keep to show that the transaction was approved. Widgets Inc. would then generate a similar receipt, which would be returned to the consumer for the on-line order placed. Note, this implies that the original request was submitted using the TAP protocol.

The idea was to take these certificates and make them short-lived. In other words, they would expire soon after their intended use. Using short-lived certificates would therefore require minimal key management and protections. What needed to be developed was an infrastructure that could deliver certificates both efficiently and securely. Under X.509-based framework, both identity and certification are carried out by a certificate authority. Under the scheme of short-lived certificates, these two processes would have to be separated. The system would need to consist of (1) a registration directory server-which contains all the named entities and their credentials, (2) a registration authority-which would be similar to the process of applying for certificates under X.509 framework; and (3) a certificate server-which would be responsible for issuing short-lived certificates.

\subsection{CGI Usage}

The NetForum capabilities are provided for by the use of common gateway interfaces (CGI). Many Web administrators do not give enough consideration to information security issues, causing CGIs on Web servers to be a major source of security holes. CGIs are not inherently insecure; they must be implemented with just as much care as the server itself. CGls have gotten a "bad" reputation because many CGI scripts have security flaws. The issue with CGIs is that they do represent an opportunity for exploitable bugs. CGIs are network-based programs and can "leak" information about the host system to a hacker. CGIs that process remote (client) user input, such as contents from a form, may be vulnerable to attacks in which a remote user can "trick" the server into executing commands. Running the Web server as a user with limited privileges can limit what executable commands can be run. Running a Web server as "nobody" (default configuration) still has enough privileges to mail out the system password file, examine the network information maps, or launch a log-in session on a high-numbered port. Even if the server runs in a chroot directory, a buggy CGI can leak sufficient system information to compromise the host. Aggregating CGIs in the cgi-bin directory is good practice for the purpose of keeping a tight inventory on CGIs installed. It is usually recommended that CGIs be restricted to the cgi-bin directory 
and by setting permissions so that only the Web administrator can install the CGIs. A tightly controlled $\mathrm{Cg} i$-bin directory helps mitigate the risk of a hacker managing to create a.cgi file somewhere in the Web server's document tree, and then executing it remotely by requesting its URL.

Generally, compiled languages such as $\mathrm{C}$ are safer than interpreted languages like Practical Extraction and Report Language (Perl), which are safer than shell scripts (e.g., Awk, Bourne, C or Korn). With some caveats, the major issue with scripts and interpreter languages is that users have access to the source code. The more a hacker knows about how a code works, the more opportunities he or she has to find mechanisms to exploit the system. With compiled codes, the compiled binary is placed in the cgi-bin directory and users can not gain access to the source code. Shell scripts have another problem: scripting languages make it extremely easy to send data to system commands and capture system command output. Compiled codes require more effort to send data to system commands, so most programmers do not use this feature. Perl has some built-in features which help to mitigate some of the security issues. The following are basic rules for evaluating or developing CGI scripts:

1. The longer and more complex it is, the more likely it is to have problems.

2. Programs that read files may inadvertently violate access restrictions that have been set up, or pass sensitive system information to hackers. Programs that write files have the potential to modify or damage documents, or introduce Trojan horses to the system. Basically, avoid giving out too much information about the network architecture and Web server.

3. CGI scripts that interact with other programs on the system should be avoided. For example, a CGI script which sends e-mail in response to a form input by opening up a connection with the sendmail program is probably insecure. Sendmail is an extremely large, complex code and historically has had many bugs.

4. CGI scripts should not run with suid (set-user-id) privileges. This is a very dangerous, and scripts must have excellent reasons for doing this.

5. CGI scripts should validate user input. Checking form input is a good security practice. Never pass unchecked remote user input to a shell command.

6. CGI scripts should use explicit path names when invoking external programs. Relying on the PATH environment variable to resolve partial path names is dangerous.

Perl programs provide the majority of the functionality in NetForum. Perl permits invocation of external programs in many different ways. Perl can capture output of an external program using backticks, can open up a pipe to a program, and can invoke an external program and never return. All these constructions are considered risky if they involve user input that may contain shell metacharacters. Perl even allows calls to an external program and "lies" to it about its name. This is useful for calling programs that behave differently depending on the name by which they are invoked. Perl has a security feature called "taint" check. This feature addresses the problem of passing unchecked user variables to the shell. Any variable that is set using data from outside the program is considered tainted and cannot be used to affect anything else outside the program. Pattern matching is another mechanism used to check information provided by a remote user. The remote user provides input, which is then compared in a pattern matching operation to a list of match file names or input.

\section{NetForum Methodology}

The IT\&S objective with NetForum is to develop an Internet-based information system which utilizes national computer networks to connect participants for conducting a meeting in "electronic space." 
NetForum would connect participants in an open, adaptive forum for information exchange, and coordinate feedback in real time. The primary means of communication will be by electronic mail (email) which will be based on hypermedia (Web browsers). The methodology would require the development of three types of tools. The first tool would be the interface to allow meeting participants view the information (i.e., meeting agenda, participant input on agenda item, side-bar discussion developing supporting or dissenting information on agenda item; issues being brought forward on agenda item). The third tool type would be the Web interface, which would help the meeting facilitator collect, digest, and disseminate the information being received back to the meeting participants in real time and in a meaningful fashion.

The NetForum activities were in two areas. The first was in defining and developing the participant and the moderator interfaces with consideration of cross-platform interface issues and the NetForum interactions which can occur. The second activity was in designing the expert system that will be used to capture, store, route, retrieve, and analyze discussion comments.

The interactions to be considered under the two NetForum activities were as follows:

1. Dissemination of information about a concept, issue, and/or recommendation;

2. Collection of information from the participants on the desirability, applicability, and success of the concept and/or recommendation;

3. Automatic identification of feedback messages in terms of the addressed recommendation or concept and the feedback message type (e.g., positive, negative, illustrative example, qualification, question about, etc.);

4. Discussions among participants across organizational boundaries (lateral communication) in regard to specific concepts, issues and/or recommendations;

5. Effective mediation by NetForum staff as information resources, moderators, and rapporteurs; and

6. Synoptic view of participants' responses to and implementation of recommendations at various levels of granularity.

Collective decision making by relevant groups of participants regarding adoption of specific recommendations included:

1. Active listening and oversight by the responsible agency (NRC);

2. Incorporation of NII technology to recuice costs; and

3. Evolution of reform structure through differentiation, revision, reframing of concepts, issues and proposals, and through identification of new categories and creation of new terms.

From an organizational perspective, the NetForum architecture should support self-correcting polity through feedback from the implementation level which facilitates reorientation and innovation. Two major feedback cycles can be identified:

- Lateral feedback-Participants share experiences laterally on concepts, issues, or recommendations and their implementation; and

- Policy feedback-Successes and failures from the implementation level provide the inputs to successive rounds of policy redirection, reformulation, and innovation. 
NetForum should prove valuable to the extent that technology and design combine to encourage participants to share dialogue rather than to posture or to express opinions. In other words, the real value will be not in people's knee-jerk reactions, but rather in the case base and on-line expertise. In a broader context, the NetForum methodology can be used to explore the utility of computers and electronic communications to enable a wide-ranging organization to more effectively locate and transfer expertise and experience, and thus, to more effectively deploy institutional memory in the service of learning, adaptation, and response to change.

\subsection{NetForum Participant Interface}

NetForum uses taxonomically organized categories to capture, store, route, and retrieve information. Figure 1 is a flow chart laying out the interface for NetForum. As shown in the fgure, the participants will participate through Web browsers using electronic forms-based input with various hypermedia tools available for viewing, aggregating, and "listening" to activity in a discussion space.

\subsubsection{NetForum Expert-Based Tools}

Under NRC funding, we extended the development of tools that the on-line facilitator uses to help focus and direct discussions, harvest and summarize comments and supporting information, reformulate the information and reintroduce it to the discussion space, identify side-bar issues and determine when they should become part of the focused discussion, and identify who should be brought together to discuss significant issues arising during a NetForum. With the use of this tool the on-line facilitator will quickly enable support, sanction and views to percolate back to the NetForum participants.

Under current ARPA (DOD) funding, several artificial intelligence (AI) systems are under development for performing language recognition and verb action. Most of these AI systems reside on expensive symbolic language machines. NetForum needs to develop an expert-based system which can perform this function on less costly machines. With the coupling of form-based e-mail and expert-based systems, the required functionality of viewing, aggregating, and "listening" to a discussion session that might include 10,000 participants over a week's time is considered feasible.

\subsubsection{NetForum Functionality}

NetForum is designed around three basic types of discussion, called discussion-open, discussionrestricted, and caucus. The type of discussion is used within the codes as an environment variable which is used to link in the functionality that has been defined for that discussion type. The NetForum also has the ability to set up discussion spaces designated as demonstration, training, and testing. 


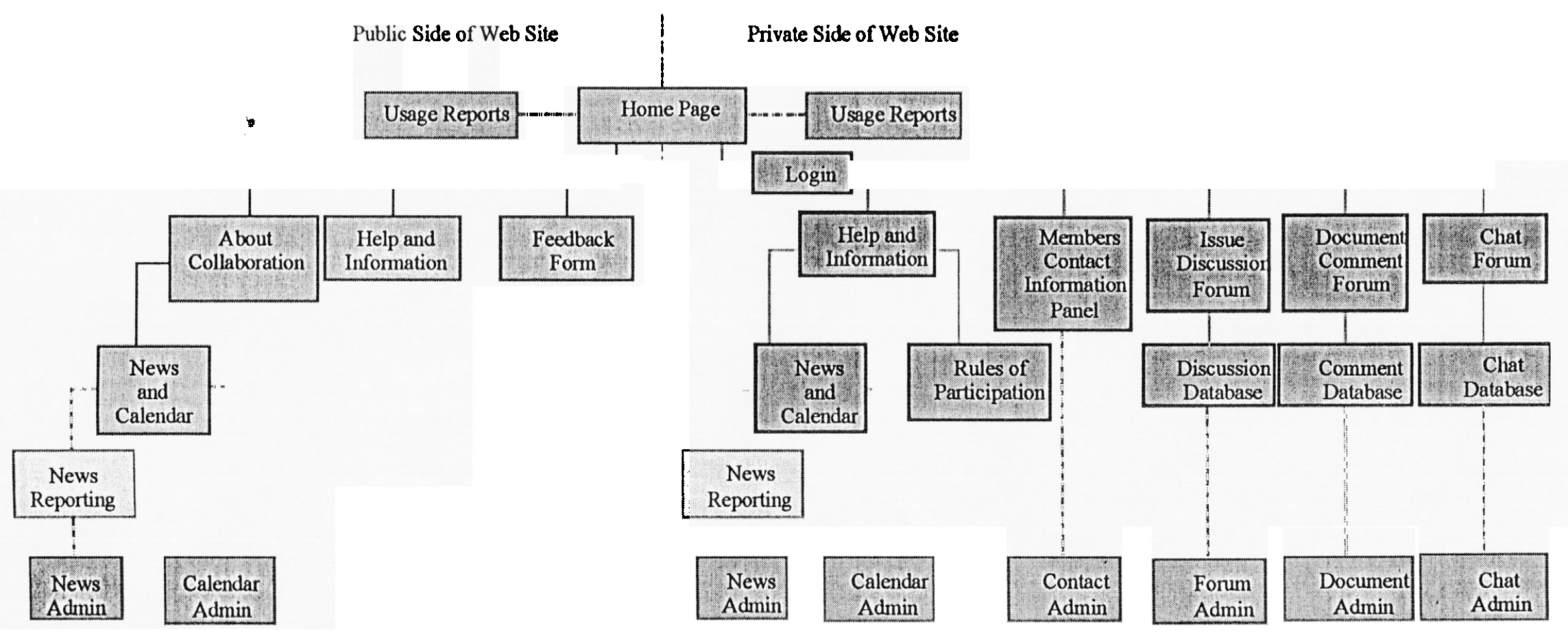

Figure 1. Flow Chart of NetForum 


\subsubsection{Discussion Type}

The NetForum discussion type is broken into two types: discussion-open and discussion-restricted. The only difference between the two is that the discussion-open allows anyone to post, while the discussionrestricted requires that a participant be "registered" to participate. The level of access control can be adjusted based on the needs of the user community. The use of access control requires that participants register to participate. With registration, participants give their e-mail address and choose a user name and password for use in the NetFonum. Their e-mail address, user name, and password are the basis for access control checking. Access control can further be restricted by system IP address. This is rather meaningless with service providers who have a single IP address or for sites with firewalls providing proxy application services. The discussion-restricted can be configured for open browsing (everyone can see but must be registered to post) or restricted browsing (cannot not see the discussion space unless they are on the access list for that NetForum space). The NetForum discussion type space can be set up to use graphical representation of the comments. The graphical representations for the comments are shown in Figure 2.

The purpose of the graphical representation of the comments is to quickly give participants an indication of how the issue is being viewed. For example, if a lot of "question" positions are being presented, then that is an indication that the issue has not been presented clearly, or that participants are needing more information to formulate opinions on the issue. It is a fairly easy means of quickly gathering an indication of what the overall position of the group is on the issue.

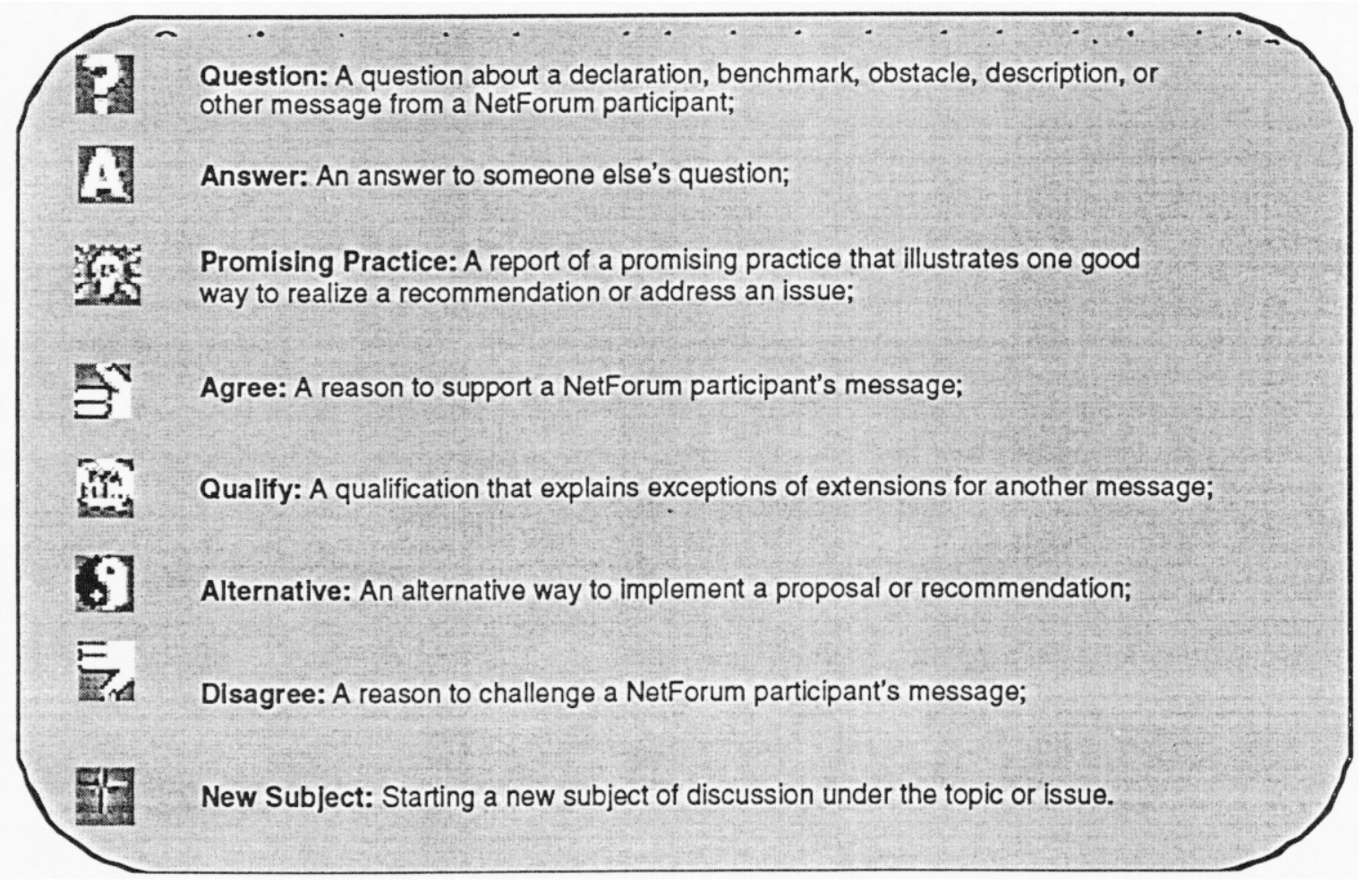

Figure 2. Graphical Representations of the NetForum Comments 
Under a discussion space defined by an issue or topic, several discussion threads can be established. Each new discussion thread under a discussion is graphically represented by the NEW icon nested to the far left of the Web page. The use of the graphical representation of the comments is handled through a software switch that allows the NetForum administrator to turn graphical comment representation on or off. The default software setting for discussion-open is with graphical representation of the links turned off while the default software setting for discussion-restricted is with graphical representation turned on. As indicated above, under an issue or topical area, the discussions are presented as threaded discussion. The nesting of the discussions gives an indication of the comment's relationship to the original comment. Figure 3 shows the nesting of the threaded discussions.

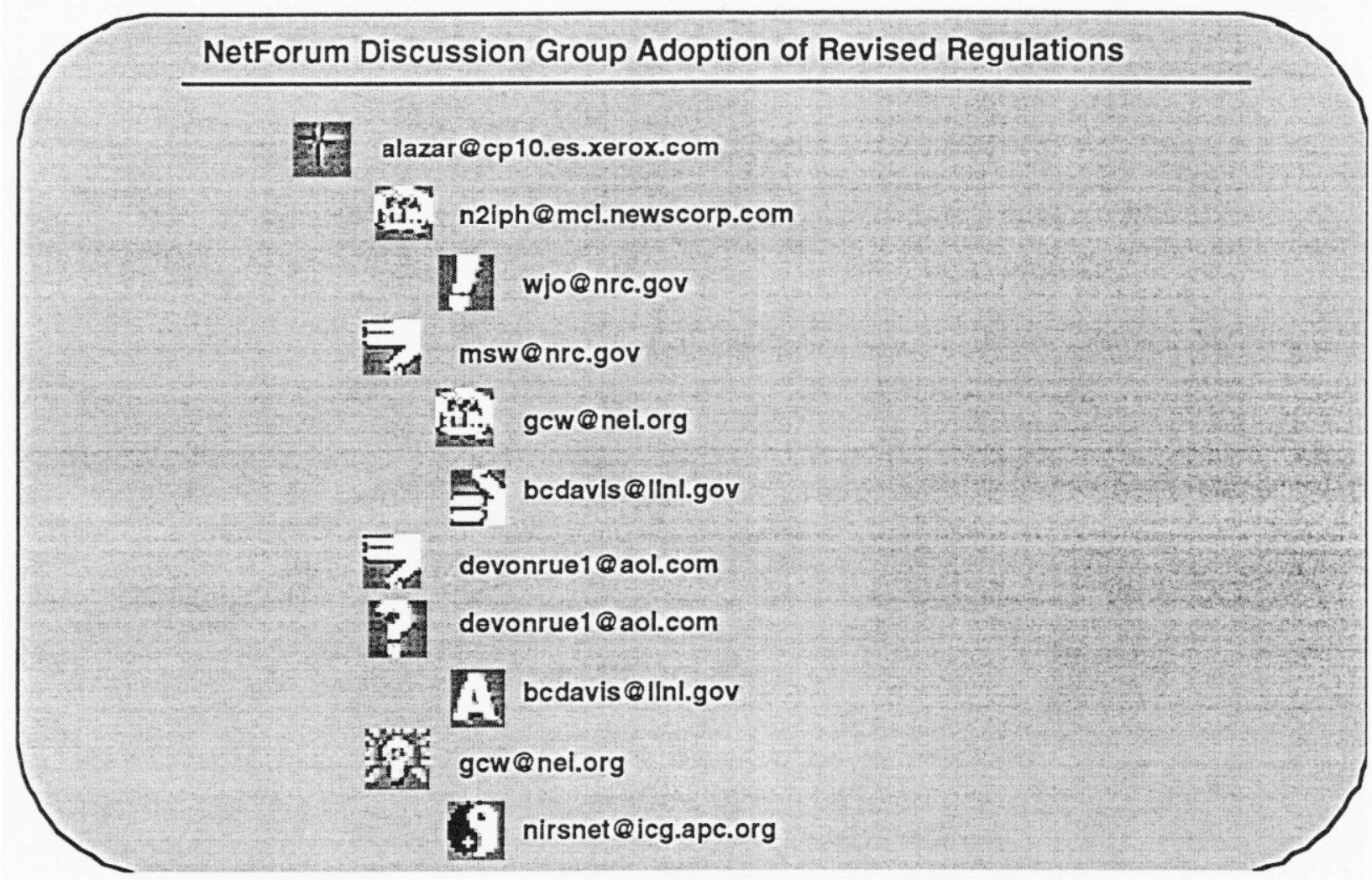

Figure 3. Nesting Relationship of Comments in a NetForum Discussion Space

At the top level of each discussion space are clickable links which look like the following:

\section{Most recent messages}

\section{Messages sorted by: [ date] [ subject] [ subject-abstract] [ position ] [ author ]}

\section{Generate a New Thread}

The "most recent message" clickable link is a "tag" link to the youngest thread (by date). Discussions may be sorted and viewed from the perspective of thread (default), date, subject, subject-abstract, position, or author. (Figure 2 is an example of a discussion sorted by thread.) The discussion comments sorted by date give the oldest message first and youngest message last, and drop the graphical 
representation of the comment in the initial page. The actual comment retains the graphical representation. The subject sort is based on alphabetical order and provides the graphical representation of the comments on the initial page. Using Figure 2 above, "Statistical vs. Probabilistic Approach" is an example of a discussion comment subject. The subject-abstract sort is the same as the subject sort except this sort provides the first 300 characters of the discussion comment message with a clickable "more" to view the remainder of the message. The final discussion comment sort available is by author. The author sorting is based on alphabetical order and drops the graphical representation of the comment.

When a user enters a discussion thread, the user can move through the comments by using clickable links to the "previous message," "next message," "previous thread," and "next thread." If the user is within the first discussion thread then the "previous thread" option will not be given.

The discussion space can be set up to be moderated or unmoderated. As with the graphical representation of the links, this option is set by a software switch. The default software setting for the discussion-open space is unmoderated, the default software setting for discussion-restricted is moderated. With either discussion space (moderated or unmoderated discussions), the comments posted to the discussion space go through a text analysis program which checks the posting against a dictionary of unacceptable words or phrases. If the message is found to have unacceptable words then the unacceptable words are highlighted and the message is sent on to the moderator (in moderated discussion) or system administrator (unmoderated discussion) for review and action. For unmoderated discussions, if the message passes the unacceptable words or phrases test, then it is immediately posted to the discussion space. As indicated, moderated discussion spaces undergo the same text analysis but are then posted to Moderator Tool for a moderator to take action on the message. The Moderator Tool is discussed in more detail below. The discussion spaces can also be facilitated. Facilitation is based on the concept of a person helping direct the conversations and calling for consensus evaluations on issues, issue ranking, and determining when to call for balloting on issues. The Facilitator Tool is discussed in more detail below.

\subsubsection{Caucus Type}

The NetForum caucus is basically the same as the discussion-restricted. The purpose of the caucus is to allow NetForum users a private discussion space. A user can request a caucus and designate who they desire to be admitted to the caucus area, and how long they desire the caucus area be open for use. The use of the caucus area is restricted to "registered" participants. The level of access control can be adjusted based on the needs of the user community. The caucus are set up to be configured for restricted browsing (cannot see the discussion space unless they are on the access list for that NetForum space).

As with the above discussion type, the NetForum caucus type space can be set up to use graphical representation of the comment. The caucus space is defined by an issue or topic; several caucus discussion threads can be established under the caucus space. Each new caucus discussion thread under a caucus is graphically represented by the "new" icon nested to the far left of the Web page. The use of the graphical representation of the comments is handled through a software switch that allows the NetForum administrator to turn graphical comment representation on or off. As indicated above, under an issue or topical area, the discussions are presented as threaded discussion. The nesting of the discussions gives an indication of the comment's relationship to the original comment. Figure 3 shows the nesting of the threaded discussions. The mechanics of the caucus type space are also as described above in Section 3.

The caucus space can be set up to be moderated or unmoderated; this option is set by a software switch. The default setting on the software switch is for unmoderated discussions. With either caucus space (moderated or unmoderated discussions), the comments posted to the caucus space go through a text analysis program which checks the posting against a dictionary of unacceptable words or phrases. If the message is found to have unacceptable words then the unacceptable words are highlighted and the message is sent on to the moderator (in moderated caucus) or system administrator (unmoderated caucus) for review and action. For unmoderated caucus, if the message passes the unacceptable words or phrases 
test, then it is immediately posted to the caucus space. As indicated, moderated caucus space undergoes the same text analysis but comments are then posted to Moderator Tool for a moderator to take action on the message. The Moderator Tool will be discussed in more detail in Section 3.2.4. The caucus spaces can also be facilitated. The facilitation is based on the concept of a person helping to direct the conversations and calling for consensus evaluations on issues, issue ranking, and determining when to call for balloting on issues.

\subsubsection{Demonstration, Training, Testing Type}

The NetForum demonstration, training, and testing type discussion space are spaces configured in whatever combination of capabilities is required for the NetForum. Where the basic differences come into play is that the consensus evaluations and ballots are preset for various times based on the Consensus Tool. The Consensus Tool will be discussed in more detail in Section 3.2.5.

\subsection{NetForum Capabilities}

As indicated, NetForum is a Web-based system, and follows standard Web convention. NetForum uses HTML, Perl, C and scripts. NetForum is a combination of capabilities defined as follows:

Forum Manager Tool-provides all the underlying functionality to process information in the NetForum discussion space.

Reference Tool-allows participants to submit documents to be reviewed and included in the NetForum.

Participant Tool-allows Internet users to register for the NetForum forum space and then participate in the forums.

Moderator Tool - allow distributed management of the NetForum discussion space to ensure relevancy and appropriateness of postings.

Consensus Tool-allows a facilitator to call for consensus evaluation of issues in the NetForum forum space, to rank issues blocking consensus, and assist in moving the discussion to consensus.

Caucus Tool-allows participants to form a group outside of the public NetForum discussion space to debate a specific issue, which may then be brought back into the NetForum discussion space.

News Tool-allows for the reporting, editing, and publishing of general NetForum information, including detailed and summary news updates of the ongoing dialogue in the NetForum discussion space.

NetDocs' capabilities include:

Distributed Authorship Tool-allows distributed document authors to submit sections of a document to the Web site so that the document is available for viewing and review by others.

Comment Tool-allows for distributed review of a document with comments per paragraph attached and traced to their authors.

Installation and Administration Tool-provides the ability to install, configure and administer the NetForum discussion space.

It should be noted that all the capabilities are anchored on "rules-of-engagement" that are laid out in the frequently asked questions (FAQs). As part of the NetForum participant registration process, users agree to follow the dialogue guidelines laid out in the FAQs. Further, the participant background information 
provided in the registration process is not publicly available (Privacy Act), while the postings in the public area of the NetForum are openly available (Freedom of Information Act). When required, nightly NetForum archives are e-mailed to the National Archive and Records Administration (NARA).

\subsubsection{Forum Manager Tool}

The Forum Manager Tool provides all the underlying functionality to process information in the NetForum discussion spaces. The Forum Manager Tool utilizes a combination of $C$ code with Perl and shell scripts and a Web-based (HTML) common software interface. This tool provides the functionality of inputting and processing the e-mail-based postings or comments, organizing the comments into the appropriate forum space, and linking to other comments. The Forum Manager Tool provides the user with the ability to sort postings by author, date, position, subject, subject-abstract, and thread. This tool also provides the notification of, and linkage to, forum consensus evaluations and ballots.

\subsubsection{Reference Tool}

The Reference Tool allows participants to submit documents to be reviewed and included in the NetForum reference space. The Reference Tool utilizes a combination of $\mathrm{C}$ code with Perl and shell scripts and a Web-based (HTML) common software interface. This tool parses documents for inclusion in the reference space of the NetForum. Supported document types are plain text (ASCII), postscript, HTML, rich text format (RTF), and word processor formats (Word Perfect, Word). The Reference Tool can be used in two modes. The first uses the paradigm of a submitter function, editor function, and librarian function. The submitter function report provides a user a means of submitting a document to NetForum for inclusion in the reference space. The editor function provides a means for a reviewer to look at the document for relevance to the forum and to check the conversion of the document's original format to HTML. The editor can bounce the document back to the submitter with comments, edit the HTML format, and add editor comments to the document, then forward it to the librarian function. The librarian function updates the reference space of the NetForum to include this document, and indexes and links the document for searching by the NetForum search engine.

\subsubsection{Participant Tool}

The Participant Tool registers users for the NetForum forum space, allowing participants to request caucus space and controlling access to the various NetForum forum spaces. The Participant Tool utilizes a combination of $C$ code with Perl and shell scripts and a Web-based (HTML) common software interface. NetForum users use the Participant Tool to submit comments in an electronic forum, read the posted comments, start new threads of discussions under a forum, indicate the graphical representation of the comment, request a caucus, and participate in consensus evaluation and ballots.

\subsubsection{Moderator Tool}

The Moderator Tool provides management of the NetForum discussion space to ensure relevancy and appropriateness of postings. The Moderator Tool utilizes a combination of $\mathrm{C}$ code with Perl and shell scripts and a Web-based (HTML) common software interface. If the NetForum discussion space is set up for moderation, then the comments must be reviewed by a moderator. The purpose of the moderator is two-fold. The first is to act as a human filter to judge the comment's acceptability. The FAQs provide the "rules of engagement;" the moderator ensures that these rules are adhered to. The second is to ensure that the discussions "stay on track." If the discussion space starts to wander off the topic of discussion, then the moderator can act to move the discussion back to the topic. Part of the Moderator Tool is a text analysis program which analyses the postings for inappropriate language. The suspect words or phrases are highlighted in bold for moderator review. The moderator has the ability to accept or reject a comment. Following acceptance the comment is posted to the electronic forum discussion space. The moderator can add comments to a posting, which appear in brackets as initialized text and are labeled "Moderator 
Comments;" the moderator can modify the comment subject; and modify the graphical representation of the comment. The Moderator Tool requires that if a moderator reflects a comment back to a participant, then a reason must be provided. The Moderator Tool also allows for multiple or cross posting of comments to other forum spaces in the NetForum. It allows the moderator to merge multiple postings. The Moderator Tool has an extension which provides the functionality of removing already-posted messages (with audit trail), modifying a message (with audit trail), splitting issues from comments, and changing nesting of discussion comments.

\subsubsection{Consensus Tool}

The Consensus Tool allows a facilitator to call for consensus evaluation of issues or a ballot, to rank issues blocking consensus, and to assist in moving the discussion to consensus. The Consensus Tool is used by a facilitator to schedule a consensus or ballot event. When a consensus or ballot event is scheduled then a calendar is generated that details for participants where consensus or ballot events are occurring and where a consensus evaluation or ballot is in the event cycle. The default schedule is for a notice to go up for 24 hours. Then the consensus evaluation opens for 48 hours. Upon closing, a summary report is generated and the issue ranking is generated. The primary function of the Consensus Tool is to generate the consensus evaluation or ballot and tabulate the consensus evaluations as the participants weigh in. It queries participants on issues blocking consensus, and then posts the ranked issues to the discussion space. Figure 4 provides the graphical representation of the consensus evaluations.

Agree [1]: An unqualified "yes" to the decision. The participant is satisfied that the decision is an expression of the wisdom of the group.

है Agree [2]: The participant is satisfied that the decision is acceptable.

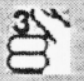

Agree [3]: The participant can live with the decision, though not particularly enthusiastic about it.

Yes, Agree [4]: The participant does not fully agree with the decision and but 4: needs to register a view about it. However, will not choose to block the decision. Is willing to support the decision because of trust in the wisdom of the group.

$E$ Disagree [5]: The participant does not agree with the decision and feels the need to stand in the way of this decision being accepted.

$E$ Disagree [6]: The participant feels no clear sense of unity in the group and feels the need for more work before consensus can be reached.

Figure 4. Consensus Evaluation in NetForum 
When participants indicate a consensus level of 4 or greater, then the Consensus Tool requires that the participant give up to three issues blocking consensus for a consensus evaluation.

For a ballot, the graphical representation used is the agree or disagree symbol (see Figure 1). The ballot does not ask for issues from participants; it only tabulates votes and generates a summary report on the ballot.

\subsubsection{Caucus Tool}

The Caucus Tool allows participants to form a discussion group outside of the public NetForum discussion space to debate specific issues, which may then be brought back into the public NetForum discussion space. Part of the Caucus Tool is a subset of the Administrator Tool; the tool creates the discussion space and posts a disclaimer. The participant requesting the caucus can specify membership, and participants use their passwords to gain access. The participant requesting the caucus specifies how long the caucus will be open. The duration of the caucus and the caucus issue are then transmitted to those participants who have been invited to join the caucus. The caucus has the same functionality as regular NetForum discussion groups.

\subsubsection{News Tool}

The News Tool allows for the reporting, editing, and publishing of NetForum-related news. When a discussion space is created, a news space for that discussion is also created. The News Tool is linked to that discussion news space and is used to generate both the "front page" summary news and the more detailed "back page" news. The News Tool has three modules. The first module is the "reporter," which is used by moderators to generate news stories for the discussion space. The second module is the "editor," which is used to create, update, delete, or archive the news stories. The third module is the "publisher," which is used to preview, publish, or archive the news. The reporter is not access-controlled; the editor and publisher are access controlled. The absence of access controls on the reporter provides the news reporter function to as many participants as desired; the editor and publisher functions are controlled by passwords. The preview function of these tools is available, but to use the modules to make permanent changes requires that the user be in the user list and have the necessary password. The News Tool allows each discussion news space be controlled by a password so that the editor and publisher function can be controlled per discussion topic.

\section{NetDocs Capabilities}

The Distributed Authorship and Comment Tools extend the functionality of the NetForum to deal specifically with discussion centered around a document. NetDocs allows threaded discussion to be anchored to paragraphs of a document. NetDocs addresses data integrity and has a formalized mechanism for resolving comment issues leading to proposed changes in the original document.

\subsection{Installation and Administration Tool (5.0)}

The Installation and Administration Tool provides the ability to install, configure, and administer NetForum and NetDocs. The installation module controls installation and configuration of NetForum. The administration module creates the new discussion topic, and creates the header and tail file format that is used to rebuild the discussion space after each comment is posted. Part of the creation of the new discussion topic is the creation of the Forum-Manager Tool, Reference Tool, Participant Tool, News Tool, Moderator Tool, Caucus Tool and the Consensus Tool. The administration module controls the granting of participants' access to the various NetForum discussion groups, and is used to close and archive a discussion topic. 


\section{GEn\&SIS Capabilities (6.0)}

The focus was on site and environmental licensing and regulatory support activities that are appropriate to GEn\&SIS. Among the key activities for siting a nuclear power plant are environmental reviews, site safety reviews (review of supporting geologic, seismologic, hydrologic, biologic, and other information), regulatory issue reviews, operating plant environmental reviews, and other site and environmental reviews as requested.

After a review of the processes and activities of the Nuclear Regulatory Commission Site and Environmental Licensing activities, the GEn\&SIS activity primarily focused on new site certification as representative of the type and nature of the information and communication processes appropriate for development for the NRC. Some aspects of this effort were also appropriate for development under the CSI project, particularly interactive geographic displays and interactive connection to analytical applications and databases.

GEn\&SIS provided the following basic functions:

- The ability to distribute and receive data through direct access via local area networked (LAN) computers, by remote access via wide area networks (WAN) (i.e., the Internet), and by standard magnetic and optical storage media.

- The ability to facilitate the acquisition of data from numerous sources in a variety of data formats. GEn\&SIS technology should encourage, through practical advantage, that various sources of information provide the agencies that information in digital format.

- The ability to provide a fast, compact display engine for the 2-D graphical display of site and environmental-related data.

- The ability to provide interactive, high-performance, real-time text and graphical display through the ACRS/ACNW conference room electronic audiovisual systems.

- The ability to support multi-point data sharing and display.

\section{Summary}

LLNL continues development of the NetForum, NetDocs, and GEn\&SIS software and the overall Internet-based collaboration methodology under funding from the Department of Energy and the Nuclear Regulatory Commission. Several Presidential Commissions and groups within the Department of Energy are currently using the software to support geographically dispersed decision making. As the software continues to be developed, several issues need to be addressed, including training in the virtual or collaborative methodology; hardware and software development, evaluation, and certification; human component; and procedures.

Specific tasks are:

1. Continue development of meeting taxonomy by categorizing existing approaches to effective meeting structure. Identify second topical implementation.

2. Continue development of hypermedia interface for meeting taxonomy. Gather reference information necessary to support second topical implementation.

3. Continue development of NetForum expert-based tool for use by discussion facilitators. 
4. Move participant comments to a discussion database.

5. Participatory decision making with larger participant pool $(100-150)$ to test NetForum expert-based tool in topical area.

6. Replace the position icons with more universally accepted symbols.

7. Develop a graphical representation of the relationship of the discussion threads.

8. Enhance NetForum expert-based tool to accommodate cross-cultural issues.

It is envisioned the more robust versions of NetForum, NetDocs, and GEn\&SIS would have added capabilities in the expert-based tool and accommodate video teleconferencing (not just the transcripts). 


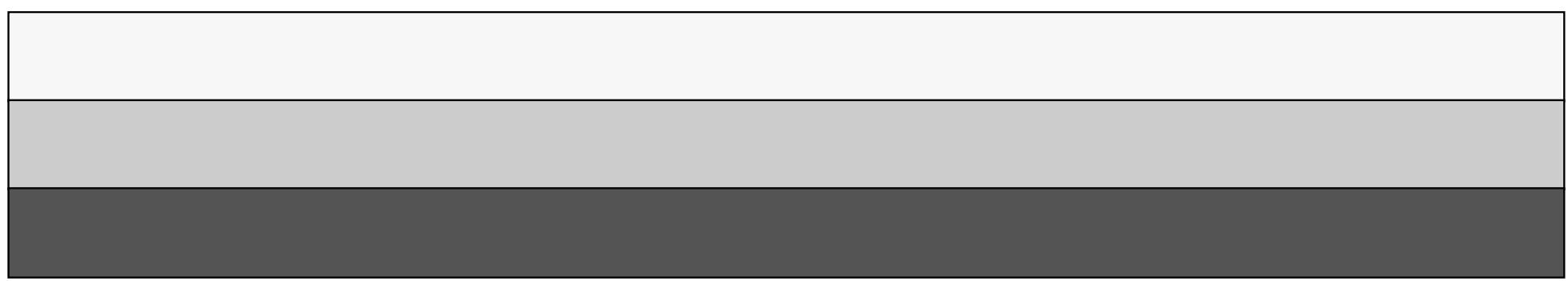

